SHEP 96/09

hep-ph/9604399

\title{
Anomalous 4-Jet Events at LEP: a Signal of Low Scale Technicolour?
}

\author{
S. F. King, \\ Physics Department, \\ University of Southampton, \\ Southampton, \\ SO9 5NH, \\ U.K.
}

\begin{abstract}
We suggest that the anomalous 4-jet events recently reported by ALEPH may be the first indication of low scale technicolour. According to our interpretation about half of the events are due to resonantly enhanced pair production of charged technipions, of mass around $55 \mathrm{GeV}$, each decaying into a pair of jets, the other half being due to standard processes. The resonant enhancement is due to a nearby technirho resonance in the mass range 150-200 GeV, leading to hugely enhanced signals at the forthcoming LEP run at $\sqrt{s}=160 \mathrm{GeV}$ since the CM energy would be closer to the peak of the resonance.
\end{abstract}


Recently ALEPH has released a preprint [1] in which they discuss some unusual 4-jet events which were previously reported in preliminary form. These data are from the LEP runs consisting of $2.8 p b^{-1}$ at $\sqrt{s} \approx 130 \mathrm{GeV}$ and $2.8 p b^{-1}$ at $\sqrt{s} \approx 136 \mathrm{GeV}$ (henceforth called LEP1.3). ALEPH studied the dijet invariant masses of 4-jet final states, and found an excess of 8 events with the sum of the two dijet masses peaked at $105 \mathrm{GeV}$. The other LEP groups do not see such a large 4-jet enhancement and it has been estimated that the probability that the 4-jet data is consistent amongst all 4 groups is about $5 \%$. However details of the ALEPH events are not consistent with QCD background, and one possibility is the pair production of new particles [1].

The angular distribution of the events is consistent with a roughly equal mixture of standard processes and scalar particle production (assuming the scalar production cross-section is large enough) [1]. Under the hypothesis that roughly $50 \%$ of the events are due to standard processes, the di-jet mass difference is consistent with either a pair of colour singlet scalar particles or a pair of coloured scalars [1]. [ Studies on electric charges further imply that the production of charged scalars is favoured over neutral scalars [1]. If the entire excess of events corresponds to the production of a pair of new particles each with a mass of about $55 \mathrm{GeV}$, and each decaying into a pair of jets then it would correspond to a production cross-section of $3.1 \pm 1.7 \mathrm{pb}$, however as we have seen it is more likely that the new physics cross-section is about half this value. Since no events contain $\bar{b} b \bar{b} b$, the effect cannot be due to $h A$ production, since both the CP-even Higgs $h$ and the CP-odd Higgs $A$ are expected to decay into $\bar{b} b$, and in any case the expected cross-section would only be about $0.5 \mathrm{pb}$ and neutral

\footnotetext{
${ }^{1}$ Neither is compatible with the angular distribution by itself, but a cocktail of $1 / 3$ standard processes plus $2 / 3$ scalar particle production is consistent with a $25 \%$ statistical error in these proportions.

${ }^{2}$ Again some fraction of the events must be due to standard processes to achieve compatibility with the di-jet mass difference. In the case of equal mass colour singlets $70 \%$ of the events are required to be due to standard processes with a large error in excess of $25 \%$. For coloured scalars or $10 \mathrm{GeV}$ mass split colour singlets the fraction of standard processes required is $30 \%$ or $25 \%$ respectively with similar errors.
} 
scalars are not favoured.

Are the events consistent with charged Higgs $H^{+} H^{-}$production? As stated, charged scalars are preferred over neutral scalars, and in this case the 4 -jet events are consistent with the decays $H^{+} \rightarrow c \bar{s}, H^{-} \rightarrow \bar{c} s$, which are one of the common decay modes of charged Higgs bosons, although no strangeness enhancement due to $K_{S}$ production is seen. 3 The other decay mode of charged Higgs is $H^{+} \rightarrow \tau^{+} \nu_{\tau}$, $H^{-} \rightarrow \tau^{-} \overline{\nu_{\tau}}$ which is not observed. For a tau branching fraction in excess of $50 \%$, the 95\% confidence level upper limit is $1.2 c \bar{s} \bar{c} s$ events [1], so the absence of such decays seems to count against the charged Higgs hypothesis, as does the expected smallness of the cross-section of about $0.4 \mathrm{pb}$ for $55 \mathrm{GeV}$ equal masses. Assuming that roughly a half of the excess of events is due to charged Higgs the theoretical cross-section still looks too small compared to the measured cross-section.

In this paper we shall explore the possibility that ALEPH are pair producing equal mass charged technipions of mass around $55 \mathrm{GeV}$ which decay predominantly into $c \bar{s} \bar{c} s$ 4-jet final states. We shall assume that roughly one half of the ALEPH 4-jet excess is due to this process, and that the other half is due to standard processes (QCD). The production rate will be enhanced by nearby technirho resonances in the mass range 150-200 GeV. In conventional technicolour (TC) the TC confinement scale $\Lambda_{T C} \sim 500 \mathrm{GeV}$ so that the technirho has a mass of about $1 \mathrm{TeV}$ [2] which is too high for the purpose of enhancing the technipion production rate. However recently we discussed the phenomenology of an $S U(2)_{T C}$ technicolour model with a low technicolour confinement scale $\Lambda_{T C} \sim 50-100 \mathrm{GeV}$ [3] the tiscussed charged technipion production, with the production cross-section resonantly enhanced by the presence of a light technirho resonance [3]. We now return to this idea in the light

\footnotetext{
${ }^{3}$ Only $3 K_{S}$ mesons are seen compared to $2.7 \pm 1.4$ expected from a normal flavour mix and $5.3 \pm 1.6$ from $c \bar{s} \bar{c} s$ final states [1]. A better fit is achieved by assuming half the events are due to scalar particles which decay into $c \bar{s} \bar{c} s$.

${ }^{4}$ We define $\Lambda_{T C}$ to be equal to half the mass of the lowest lying vector resonance.
} 
of the ALEPH 4-jet excess. As we shall see, assuming that ALEPH are seeing such a signal, the implications of this scenario for the LEP run in June of this year at $\sqrt{s}=160 \mathrm{GeV}$ (henceforth called LEP 1.6) are so great that it as well that our experimental and phenomenological colleagues are made aware of them now. This is our principal motivation for writing this paper at the present time.

The basic production mechanism we suggest is:

$$
e^{+} e^{-} \rightarrow \gamma^{*}, Z^{*} \rightarrow \rho_{T C}^{*} \rightarrow \pi_{T C}^{+} \pi_{T C}^{-}
$$

The partial width of the technirho 9 into a single pair of charged technipions is given by a scaling argument as,

$$
\Gamma\left(\rho_{T C} \rightarrow \pi_{T C}^{+} \pi_{T C}^{-}\right) \approx \frac{m_{\rho_{T C}}}{m_{\rho}} \frac{\beta_{\pi_{T C}}^{3}}{\beta_{\pi}^{3}} \Gamma\left(\rho \rightarrow \pi^{+} \pi^{-}\right)
$$

where $\beta_{\pi(T C)}=\left(1-\frac{4 m_{\pi(T C)}^{2}}{m_{\rho(T C)}^{2}}\right)^{1 / 2}$ is the relevant phase space factor where $m_{\pi_{T C}}$ is the technipion mass. In this case the full width of a technirho is approximately equal to the partial width into technipions,

$$
\Gamma_{\rho_{T C}} \approx \Gamma\left(\rho_{T C} \rightarrow \pi_{T C}^{+} \pi_{T C}^{-}\right)
$$

It is clear that the presence of a light technirho will serve to resonantly enhance the production of charged technipions relative to that of charged Higgs bosons of the same mass. According to VMD arguments (see later), in the resonance region we expect this enhancement to be given by a factor $R$ where

$$
R=\frac{\sigma\left(e^{+} e^{-} \rightarrow \pi_{T C}^{+} \pi_{T C}^{-}\right)}{\sigma\left(e^{+} e^{-} \rightarrow H^{+} H^{-}\right)} \approx \frac{m_{\rho_{T C}}^{4}}{\left[\left(s-m_{\rho_{T C}}^{2}\right)^{2}+\Gamma_{\rho_{T C}}^{2} m_{\rho_{T C}}^{2}\right]}
$$

where $\sigma\left(e^{+} e^{-} \rightarrow H^{+} H^{-}\right)$is the usual production cross-section for charged Higgs bosons in a two-Higgs doublet model. This expression is valid for any charged technipion mass (assumed to be approximately equal to the corresponding charged Higgs mass).

\footnotetext{
${ }^{5}$ In fact there may be more than one technirho, however for simplicity we shall assume a single technirho resonance.
} 
In Table 1 we estimate the enhancement ratio $R$ as a function of the technirho mass at LEP1.3 and give predictions for the expected enhancement at LEP1.6. For example suppose we require $R \approx 10$ in order to account for the ALEPH data. Then according to Table 1 such an enhancement implies that $m_{\rho_{T C}} \approx 160 \mathrm{GeV}$ and $\Gamma_{\rho_{T C}} \approx 15 \mathrm{GeV}$. In this example the estimated technirho mass is exactly equal to the CM energy of LEP1.6 which will be sitting at the peak of the technirho resonance with

$$
R(\sqrt{s}=160 G e V) \approx \frac{m_{\rho_{T C}}^{2}}{\Gamma_{\rho_{T C}}^{2}} \approx 114
$$

corresponding to a production cross-section of $55 \mathrm{GeV}$ charged technipions of about 63pb! Of course the exact enhancement factor is poorly determined at LEP1.3 so in Table 1 we have considered technirho masses in the range 150-200 GeV. In all cases it is clear that LEP1.6 will always be closer to the peak of the resonance leading to much greater enhancements than those presently observed. Table 1 is only meant as a rough guide to the expected rates and if LEP1.6 does see a strong enhancement of the 4-jet rate it will be a straightforward task to use Eqs.214 to determine accurately the mass and width of the technirho.

\begin{tabular}{|c|c|c|c|}
\hline$m_{\rho_{T C}}(\mathrm{GeV})$ & $\Gamma_{\rho_{T C}}(\mathrm{GeV})$ & $R(\sqrt{s}=133 \mathrm{GeV})$ & $R(\sqrt{s}=160 \mathrm{GeV})$ \\
\hline 150 & 11 & 20 & 41 \\
\hline 160 & 15 & 10 & 114 \\
\hline 170 & 18 & 6 & 41 \\
\hline 180 & 21 & 4.5 & 17 \\
\hline 200 & 28 & 3 & 7 \\
\hline
\end{tabular}

Table 1: Predictions of the model for technirho masses in the range 150-200 GeV. For each mass value $m_{\rho_{T C}}$ we tabulate the width $\Gamma_{\rho_{T C}}$ (calculated from Eq.2) and the ratio $R$ (calculated from Eq.(4) of the charged technipion production cross-section to the charged Higgs production crosssection for LEP1.3 and LEP1.6 energies. Absolute values of cross-sections may easily be obtained from $R$ by noting that the tree-level cross-section for $55 \mathrm{GeV}$ charged Higgs production at LEP1.3 (LEP1.6) is $0.43 \mathrm{pb}(0.55 \mathrm{pb})$. 
In order to understand the above VMD results, and obtain other predictions it is necessary to give some details of the model. Here we shall present a stripped down version of the low scale technicolour model introduced in ref. [3] which is sufficient for our purposes. In the complete model there are three "generations" of technifermions, one of which is very heavy and breaks electroweak symmetry, and two of which are light and accessible to LEP [3]. Here we will retain the single Higgs doublet $H$ of the standard model, and consider only one of the two light generations of technifermions for simplicity. The model is based on the gauge group

$$
S U(2)_{T C} \otimes S U(3)_{C} \otimes S U(2)_{L} \otimes U(1)_{Y}
$$

where we have added to the standard model gauge group a new confining QCDlike gauge group $S U(2)_{T C}$ which is asymptotically-free and confines at $\Lambda_{T C}=75-$ $100 \mathrm{GeV}$. We assume that the usual three families and Higgs doublet are TC singlets, and transform in the usual way under the standard part of the gauge group. In the stripped down low scale technicolour model here we shall introduce only one doublet of technifermions $(p, m)$, which have electric charges $(1 / 2,-1,2)$, respectively, and which transform in an anomaly-free way under the full gauge group as,

$$
\begin{aligned}
t_{L}=\left(\begin{array}{c}
p_{L} \\
m_{L}
\end{array}\right) & \sim(2,1,2,0) \\
p_{R} & \sim(2,1,1,1 / 2) \\
m_{R} & \sim(2,1,1,-1 / 2)
\end{aligned}
$$

It is worth emphasising that this is not an extended TC model since the technifermions have no additional couplings to the ordinary quarks and leptons beyond those of the standard model.

In the present model electroweak symmetry is broken predominantly by the usual Higgs vacuum expectation value (VEV) $v$, since $\Lambda_{T C}<v$. We assume that the technifermions have Yukawa couplings to Higgs doublet of the form $\lambda_{p} \overline{p_{R}} H^{c \dagger} t_{L}, \lambda_{m} \bar{m}_{R} H^{\dagger} t_{L}$ resulting in technifermion current masses $m_{p}=\lambda_{p} v, m_{m}=\lambda_{m} v$. We expect TC condensates to form near the TC confinement scale but now there is a vacuum alignment 
problem which depends on the technifermion current masses. The TC gauge forces tend to favour the chiraly invariant condensate of the form $\left\langle\bar{t}_{L}^{\bar{c}} t_{L}+t_{R}^{\bar{c}} t_{R}>\neq 0\right.$, while the current mass terms prefer the chiral symmetry breaking condensates of the form, $<\overline{t_{L}} t_{R}+\overline{t_{R}} t_{L}>\neq 0$. 9 We assume here that the latter condensates form, leading to global chiral symmetry breaking in the TC sector,

$$
S U(2)_{L} \otimes S U(2)_{R} \rightarrow S U(2)_{L+R}
$$

yielding a triplet of technipions $\pi_{T C}^{ \pm, 0} \sim \bar{t} \sigma^{ \pm, 3} \gamma_{5} t$, with $<0\left|j_{\mu 5}^{a}\right| \pi_{T C}^{b}>=i f_{T C} q_{\mu} \delta^{a b}$, where $f_{T C} \sim 15-20 \mathrm{GeV}$, and the current is $j_{\mu 5}^{a}=\bar{t} \gamma_{\mu} \gamma_{5} \sigma^{a} t$.

The current masses of the technifermions break the chiral symmetry of the technidoublet resulting in a physical technipion mass analagous to the way in which the physical pion mass results from explicit quark masses. The technipion mass $m_{\pi_{T C}}$ may be estimated by scaling up the usual result for the ordinary pion mass $m_{\pi}$,

$$
m_{\pi_{T C}}=m_{\pi} \sqrt{\left(\frac{m_{p}+m_{m}}{m_{u}+m_{d}}\right) \frac{f_{T C}}{f_{\pi}}} .
$$

Assuming that the mass of the technipions is around $50-60 \mathrm{GeV}$ we thus deduce that $m_{p}+m_{m} \sim 10 \mathrm{GeV}$. The charged technipions $\pi_{T C}^{ \pm}$will decay predominantly via virtual $\mathrm{W}$ exchange, analagous to ordinary charged pion decay. Thus for example the width into leptons is given by,

$$
\Gamma\left(\pi_{T C}^{ \pm} \rightarrow l^{ \pm} \nu_{l}\right)=\frac{f_{T C}^{2}}{f_{\pi}^{2}} \frac{m_{l}^{2}}{m_{\mu}^{2}} \frac{m_{\pi_{T C}}}{m_{\pi}} \frac{1}{\left(1-\frac{m_{\mu}^{2}}{m_{\pi}^{2}}\right)^{2}} \Gamma\left(\pi^{ \pm} \rightarrow \mu^{ \pm} \nu_{\mu}\right) .
$$

The largest such decay channels are thus $c s$ and $\tau \nu_{\tau}$ (the $c b$ channel is suppressed by $\left.V_{c b}\right)$. The $c s$ channel is responsible for some of the 4 -jet events observed by ALEPH. Since the decay goes via the $W$ couplings the $\tau \nu_{\tau}$ channel should also be observed at about $1 / 3$ the rate of the $c s$ channel due to colour, although there are calculational

\footnotetext{
${ }^{6}$ In the complete model it is likely that both sorts of condensates form, with the first lighter generation of technifermions yielding chirally invariant condensates, and the heavier second generation yielding chiral symmetry breaking condensates.
} 
uncertainties due to QCD corrections (and the charm mass). In addition there may be substantial model dependence if the simple TC model presented here is extended. Nevertheless one would expect that the $\tau \nu_{\tau}$ decay channel should have a significant branching fraction at some level and should sooner or later be observed.

In addition to the charged technipions $\pi_{T C}^{ \pm}$the model clearly also predicts neutral technipions $\pi_{T C}^{0}$ of mass around $55 \mathrm{GeV}$. The neutral technipions $\pi_{T C}^{0}$ decay via virtual $\mathrm{Z}$ exchange, with the largest partial width,

$$
\Gamma\left(\pi_{T C}^{0} \rightarrow \bar{b} b\right) \approx 3\left(\frac{m_{b}^{2}}{m_{l}^{2}}\right) \Gamma\left(\pi_{T C}^{ \pm} \rightarrow l^{ \pm} \nu_{l}\right) .
$$

$\pi_{T C}^{0}$ will also decay into two photons via a chiral symmetry suppressed anomalous $\pi_{T C}^{0} \gamma \gamma$ coupling [3]. Neutral technipions may be produced singly at LEP via the production mechanism:

$$
e^{+} e^{-} \rightarrow \gamma^{*}, Z^{*} \rightarrow \rho_{T C}^{*} \rightarrow \pi_{T C}^{0} \omega_{T C}^{*}
$$

The techniomega couples to charged leptons $l^{ \pm}$in the complete model [3] leading to signatures of the type: $\bar{b} b l^{+} l^{-}$or $\gamma \gamma l^{+} l^{-}$where the mass of the $\bar{b} b$ or $\gamma \gamma$ is equal to that of the $\pi_{T C}^{0}$, and the mass of the $l^{+} l^{-}$peaks near the techniomega mass. The precise rate is difficult to estimate [3].

Apart from the low-scale technipions, the technidoublet $t=(p, m)$ will give rise to the technivector mesons $V$ mentioned above, which are analagous to the QCD vector resonances. For example we may expect a $J^{P C}=1^{--}$technirho isotriplet $\rho_{T C}^{ \pm, 0}$ and techniomega isosinglet $\omega_{T C}^{0}$ with masses in the range $150-200 \mathrm{GeV}$. The vector masses are given by scaling up the ordinary $\rho$ and $\omega$ mass $m_{\rho_{T C}, \omega_{T C}} \approx m_{\rho, \omega} \frac{f_{T C}}{f_{\pi}}$. The technidoublet $t$ has photon and $\mathrm{Z}$ couplings,

$$
A_{\mu} \bar{t} \gamma^{\mu} Q t+Z_{\mu} \bar{t} \gamma^{\mu}\left(\Gamma_{V}+\Gamma_{A} \gamma_{5}\right) t
$$

where

$$
Q=e \frac{\sigma^{3}}{2}, \quad \Gamma_{V}=\frac{e}{\tan 2 \theta_{w}} \frac{\sigma^{3}}{2}, \quad \Gamma_{A}=\frac{-e}{\sin 2 \theta_{w}} \frac{\sigma^{3}}{2} .
$$


Using vector meson dominance (VMD) arguments, combined with scaling-up arguments, we write,

$$
\bar{t} \gamma^{\mu} \frac{\sigma^{a}}{2} t \rightarrow \frac{m_{\rho_{T C}}^{2}}{g_{\rho_{T C}}} \rho_{T C}^{a \mu}
$$

where $g_{\rho_{T C}} \approx g_{\rho}$, and $g_{\rho}=\sqrt{12 \pi}$. This implies the following technirho $\square$ couplings involving the photon and $\mathrm{Z}$

$$
\frac{m_{\rho_{T C}}^{2}}{g_{\rho_{T C}}} \rho_{T C}^{0, \mu}\left[e A_{\mu}+\frac{e}{\tan 2 \theta_{w}} Z_{\mu}\right] .
$$

A similar line of reasoning leads to technirho couplings to technipions given by

$$
-g_{\rho_{T C}} \epsilon_{a b c} \rho_{T C}^{0, a, \mu} \pi_{T C}^{b}\left(\partial^{\mu} \pi_{T C}^{c}\right)
$$

The above VMD results were in fact used to give the earlier prediction for $R$ in Eq.⿴囗十 due to the mechanism in Eq.11.

One may wonder about the contribution of charged technipions to $b \rightarrow s \gamma$. While charged Higgs of $55 \mathrm{GeV}$ would give a very large contribution to $b \rightarrow s \gamma$, in this model technipions of a similar mass do not contribute to this process. This is because the technifermions in this model have no direct couplings to ordinary fermions (i.e. no extended technicolour couplings) other than via the standard model Higgs doublet and $W, Z$ couplings. However one would expect the charged technipions to be produced in the top quark decays at the Tevatron $t \rightarrow \pi_{T C} b$ at some level.

Also one may be concerned about the constraints on technicolour from high precision tests of the standard model. At LEP1 energies the technirho will contribute to the oblique corrections to the photon and Z propagators. For example the contribution to the S parameter [4] for a single technidoublet is naively estimated to be $S \approx 0.3\left(\frac{2}{3}\right) \approx 0.2$. . For two technidoublets our naive estimate satisfies the phenomenological constraint [6] $S<0.38(0.46)$ at $90 \%(95 \%)$ CL, but this estimate is

\footnotetext{
${ }^{7}$ Note that according to Eq.14 the isosinglet techniomega has no direct coupling to the photon or $Z$ in this model.

${ }^{8}$ Note that the TC contribution depends on scale independent ratios like $\frac{f_{T C}}{m_{\rho_{T C}}}$ so is independent of the fact that the technicolour scale (and the technirho mass) is low, assuming that the technirho
} 
likely to be unreliable since the first generation technidoublet is not assumed to form QCD-like condensates. For three technidoublets we must begin to appeal to such unreliability of the estimates.

Finally it should be pointed out that there are other models in the literature which also have a low TC scale, for example ref.[7]. These authors were concerned with signals at hadron colliders and pointed out that the technirho could be produced at the Tevatron and could decay into a technipion $\pi_{T C}^{ \pm}$plus a longitudinally polarised $W_{L}$, yielding a characteristic signature. Although such decays are suppressed relative to the two technipion decays by a factor $\left(f_{T C} / 245 \mathrm{GeV}\right)^{2}$, they would dominate if the technipions are heavier than half the technirho mass. However this condition is clearly not fulfilled here since the technipions have masses around $55 \mathrm{GeV}$ while the technirho has a mass in the range 150-200 GeV. Therefore such decays will have a very small branching fraction at the one per cent level and are unlikely to be observable at the Tevatron, although interestingly such decays may be observed in the high statistics clean environment of LEP1.6.

To summarise, we have proposed that approximately a half of the anomalous 4-jet events seen by ALEPH at LEP1.3 are due to resonantly enhanced charged technipion production, involving charged technipions. If this interpretation is correct then we should expect even more enhancement in the rate at LEP1.6 since the CM energy will be closer to the pole of the technirho, as is clearly seen in Table 1. The discovery of a light technirho would be the biggest bombshell in high-energy physics since the discovery of the $J / \psi$, and we look forward to the next LEP runs in June with some excitement.

\section{Acknowledgement}

is much heavier than the $Z$ boson. However our estimate relies on VMD and large $N_{T C}$ scaling arguments in order to estimate the contribution to dispersion relations, and such may be unreliable in TC theories $[5]$. 
I would like to thank A. Vayaki and P. Janot for useful communications.

\section{References}

[1] "Four-jet final states production in e+e- collisions at centre-of-mass energies of 130 and 136 GeV", The ALEPH collaboration, CERN PPE/96-52.

[2] For a recent review of technicolour see S. F. King, Rep. Prog. Phys. 58 (1995) 263.

[3] S. F. King, Phys. Lett.B314 (1993) 364.

[4] M. Peskin and T. Takeuchi, Phys. Rev. Lett. 65 (1990) 964; ibid Phys. Rev. D46 (1992) 381; R. N. Cahn and M Suzuki, Phys. Rev.D44(1991)3641.

[5] K. Lane, Proc.XXVII Int.Conf. on High Energy Physics, Glasgow 1994, p.543, ed.P.Bussey and I. Knowles.

[6] P. Langacker and J. Erler, in the Review of Particle Properties, Particle Data Group, Phys. Rev.D50(1994)1312.

[7] E. Eichten and K. Lane, Phys. Lett.B222 (1989) 274. 